\title{
Integrating Human Performance Measures into Space Operations: Beyond Our Scheduling Capabilities?
}

\author{
Jessica J. Marquez \\ NASA Ames Research Center \\ M/S 262-2, Bldg. 262, Rm. 132, P.O. Box 1 \\ Moffett Field, CA 94035 \\ 650-604-6364 \\ Jessica.J.Marquez@nasa.gov
}

\begin{abstract}
Current planning and scheduling software tools for International Space Station (ISS) support different flight controller teams as they plan daily space operations. Planning and scheduling tools capabilities include integrating digitized ISS state inputs, evaluating their expected future states, and propagating them over time. Extensive, custom-made computational models of operations, of objectives, and of operational constraints help ISS flight controllers identify where scheduled events violate constraints. Based on the current capabilities of these tools, this paper proposes how human performance measures could be better integrated into planning and scheduling tools for space mission operations. Future integration of human performance measures could be applied to state inputs (in this case, the astronaut's state) and to modeling human performance operational constraints \& operational objectives (i.e., assigned activities) with parameters that are relevant to human performance measures. Gaps between the state-of-the-art for human performance modeling and planning tools for future exploration missions are identified.
\end{abstract}

\section{TABLE OF CONTENTS}

1. INTRODUCTION 1

2. SPACE OPERATIONS PlaNNING ........................1

3. Integrating Human Performance Measures into SPaCe OPERATIONS ...................4 4. DISCUSSION.........................................................6

5. ConCLUSION.......................................................8

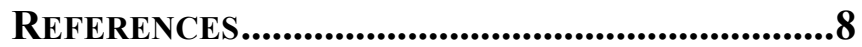

BIOGRAPHY .................................................................9

\section{INTRODUCTION}

One of the most limited resources in human spaceflight is crew time. For International Space Station (ISS) operations, the flight control team plans crew time carefully, considering what is the best time for crew to perform assigned activities. Currently, human performance measures and modeling are not tightly integrated into these planning and scheduling tools. In future exploration missions, NASA may need to integrate human performance modeling into crew scheduling tools in order to better support crew autonomy and to maximize crew time while still minimizing crew performance errors. This paper describes the current planning processes used for crew planning for ISS operations, and then proposes how human performance measures could be more highly integrated into this process.

\section{Space Operations Planning}

Space operations planning for the International Space Station (ISS) program is the current best practices for human spaceflight. ISS planning is complex; it requires the integration of many analyses from various flight controller disciplines, meeting the needs of the International Partners (IPs) and scientific payloads, while still satisfying the hundreds of ISS operational constraints. These constraints range from hardware limitations (e.g., maximum amount of shadows the solar arrays are allowed) to operationally defined planning constraints (e.g., required bi-weekly meetings between crew and flight doctors).

Figure 1 notionally describes the planning process for ISS

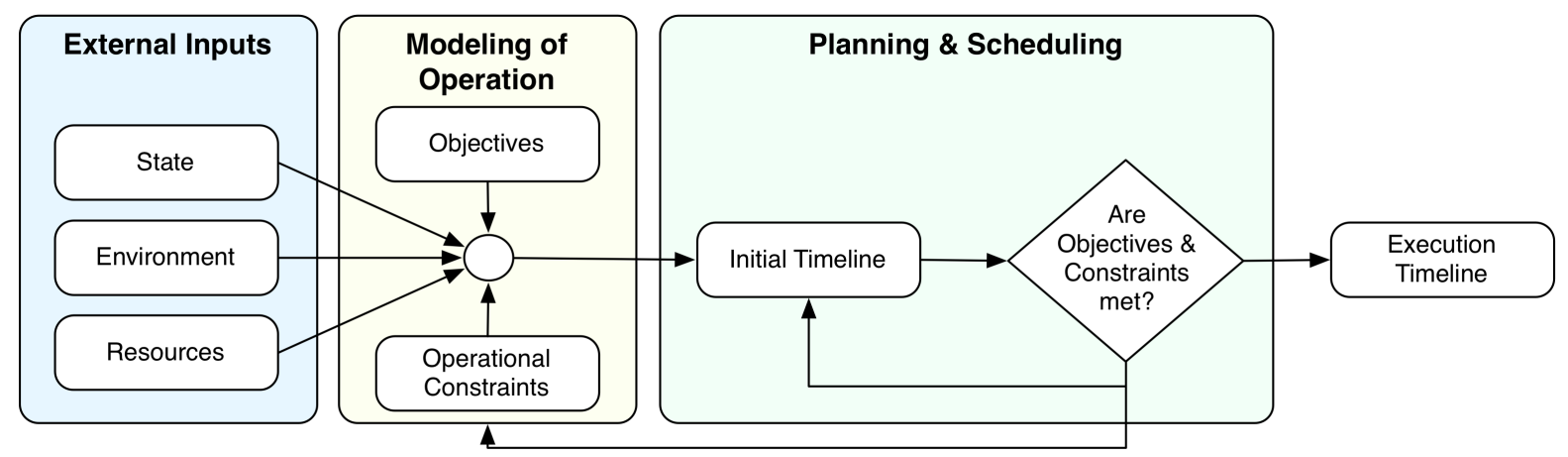

Figure 1: Notional depiction of ISS planning and scheduling process 
space operations. This illustration (adapted from [1,2]) captures the observed planning process for the space station vehicle, for payloads, and for crew. It is important to acknowledge that ISS space operations planning is one that is operationally feasible given the resources available to the program, and not necessarily one that is theoretically optimal. Inherently, achieving an optimal plan is limited by the fidelity and accuracy of the models for operations, constraints, and environment.

In general, the ISS planning and scheduling process starts with the state of vehicle and payloads, the predicted environment for the relevant time frame, and the available resources (e.g., number of people or power available). These serve as inputs to the integration process of modeling and analyzing operations, given the operational constraints and set of objectives for the given time frame, i.e., the operations to be completed. Detailed planning and scheduling is conducted a couple of weeks ahead of execution time. This analysis results in an initial timeline (set of activities for a specific time). Part of the planning and scheduling process is to verify that the objectives and all the operational constraints are satisfied before an official plan and schedule is published to the entire mission control flight team.

Once there is a shared plan, flight controllers can formally request changes and replanning ensues. Typically, plan changes are not requested the day of execution. Flight controllers monitor their system to verify that the planned activities are completed as expected and ISS is still operating within the required constraints. If issues arise, for instance there is some emergency or hardware anomaly [3], flight controllers take immediate action to ensure the safety of the crew and safeguard the spacecraft. If these events result in planned activities being delayed, they are rescheduled in the near future.

\section{Planning and Scheduling Capabilities}

There are many aspects of ISS space operations that are planned and scheduled [4, 5]. Among these are: extravehicular activities (i.e., EVA or spacewalks); power availability and consumption; attitude control and maneuvers; active environmental, life support, and thermal control; communication, command, and data handling; and crew and payload schedules. For each of these disciplines or domains, ISS flight controllers predict and analyze carefully a "week in the life" of the space station. Each discipline has a set of software tools to support their planning needs.

ISS power planning illustrates the capabilities that currently exist in planning and scheduling tools. For the discipline of ISS power availability and consumption, the Power Resource Officer (PRO) conducts a weeklong analysis of ISS power availability in order to determine if there is enough to support the ISS's consumption needs while still satisfying all the hardware and operational constraints. As in Figure 1, PROs must first gather all the state inputs, such as the position of the space station and the configuration of solar array joints. Environment inputs include the expected position of the sun relative to ISS orbital dynamics. The main resource for ISS power planning is power availability, which is driven by the ISS power configuration, including the number of solar arrays available and relationship between power converter units, power controllers, and batteries.

Once these inputs are gathered, the PROs must model the required objectives (see Figure 1) - in this case, the items that are consuming power. There are items that require constant power (like basic life support for crew) while others, such as specialized payloads, are only turned on (or off) when necessary. In this discipline, the required objectives are modeled as scheduled activities that draw a calculated amount of power for a particular length of time. Additionally, other objectives come in as scheduled activities that have impacts on power planning. For example, during an EVA or a ISS reboost event, the solar arrays must be locked into place, as opposed to constantly rotating, tracking the sun. This information is integrated into the many complex models of operation the PROs (and other flight controller) disciplines have their disposal. Power analysis is computed through various custom-made models that are all integrated through a single software user interface, Power Planning Analysis Tool (PLATO) [5].

Once the power availability is estimated as a function of time, the PROs need to verify that there is enough for the expected power consumption, also modeled in PLATO. This is the planning and scheduling process described in Figure 1. During the analysis, the responsible flight controller needs to verify that not only all the items that require power have enough, but also the hardware and operational constraints are met. Often, the PRO needs to shift the ISS power configurations in order to satisfy demand, i.e., providing appropriate electrical power to subsystems that require it. They analyze various configurations with their planning software tools; for example, they might schedule to turn off certain nonessential payloads or divert electric power from one converter unit to another at specific times. In turn, these loading profiles exercise the hardware limits, like the minimum battery discharge allowed or the maximum power load per solar array permitted. They leverage their planning and scheduling tool, PLATO, to identify exactly when these types of violations occur (Figure 2). When the power needs are not met by the availability, the mission control team has to change the required objectives or relax some constraint. Once the flight controllers have arrived to a satisfactory plan, this is shared with the rest of the mission control team and the changes to power configuration are executed during flight. 


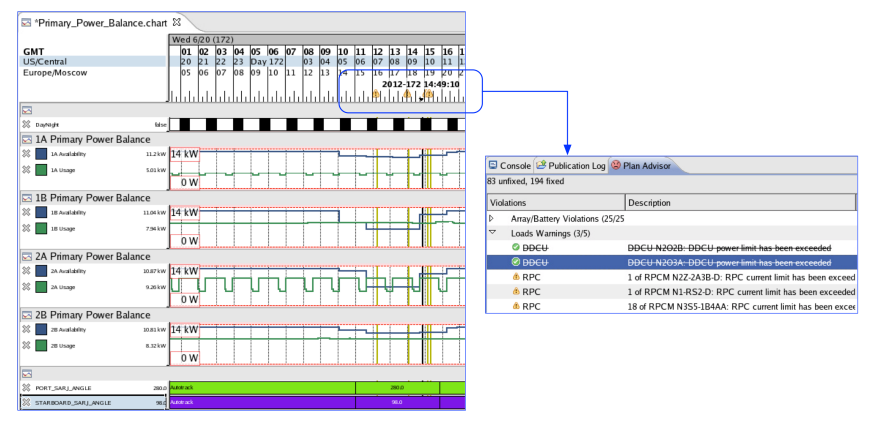

Figure 2: PLATO graphs of predicted power availability versus usage with violation warnings

In summary, ISS power planning exemplifies the current planning and scheduling capabilities. Flight controllers are able to collect digitized ISS state inputs and evaluate their expected future states, propagated in time. They are able to leverage extensive, custom-made computational models of operations, of objectives, and of operational constraints in order to conduct planning analysis. Software helps the flight controllers identify time periods and configurations that violate constraints, and they use these same planning software tools to schedule key activities that permit ISS operations within the defined constraints.

\section{ISS Crew Planning}

Planning and scheduling for ISS is multifaceted and detailed as it centers on engineering and hardware models and analyses. ISS crew planning and scheduling, while similar, differs from other ISS planning because the crew operations is not modeled at the same fidelity. Crew operations are mostly modeled through crew time, as it is the most constraining resource. Each activity, or task, that the crew must perform is characterized in a several ways, though the main attributes relevant to human performance are: number of crewmembers assigned to it, its duration, and type of activity. The type of activity is a simple grouping, for example, maintenance tasks or part of a docking/undocking event. The key difference between these groupings is if this activity is part of ISS work or if it is routine. Routine tasks are those like exercising, eating, or sleeping. Only activities labeled in non-routine groups count towards work-hours aboard ISS.

Aside from crewmembers' time, there are many program requirements, planning and operational constraints that must be met during crew planning. (Planning constraints differ from flight rules since the latter govern real-time operations.) Program requirements are events such as docking/undocking of visiting space vehicles, emergency EVAs or required scientific experiments. Many of planning constraints are documented in the ISS Generic Groundrules and Constraints (GGr\&C) [6]. The planning constraints are varied. For example, the allowable maximum number of work-hours for each consecutive workday is specified. The GGr\&C also specifies the number of days off that crew must have, including those preceding and following visiting vehicles and EVA events. Another example is the required meetings to be scheduled with ground (e.g., number of times crew must talk to their flight doctors).

While activity duration and type of activity are relevant to human performance modeling, other parameters related to the crews' assigned task are modeled. Tasks that are assigned for crew to execute include further modeling information, such as resource needs. A simple example is exercise. These activities require particular exercise machines (like treadmill or resistive device) and are modeled as such. Once that exercise activity is scheduled for one crewmember, a similar activity requiring the same exercise device cannot be scheduled for another crewmember. Likewise, activities that require particular communication bandwidth are modeled in a similar manner. Temporal relationships between crew tasks are modeled as well; typically, these activities have a natural grouping, like a sequence of activities gearing up to a particular event. Activities can also be modeled to affect resources. As discussed, crew time is the main resource modeled through duration, but the amount of power and data usage per activity is also computed.

Ops Planners (and their flight control team) are responsible for integrating all assigned crew activity for an ISS expedition. They use Score as their main planning and scheduling tool [5]. Score has similar scheduling and planning capabilities like PLATO, though Score emphasizes integrated activity resource modeling while PLATO has more sophisticated planning analysis functions. Score receives a variety of digitized ISS state inputs as well as resource calculations from several other flight controller disciplines. With Score, planners can easily schedule and reschedule activities, model operational constraints, and integrate the IPs program requirements. Furthermore, Score identifies periods in the timeline where the operational constraints have been violated, facilitating scheduling conflict resolutions.

Since this paper focuses on current space planning and scheduling capabilities and integration with human performance planning, it is worth noting a few key differences (which will be expanded on in subsequent sections) between system-level space operations planning and planning for optimized human performance. While spacecraft systems operations are modeled, human performance is not modeled per se within the ISS Ops Planner planning and scheduling tools, with the exception of time on task. If human performance is modeled in detail, it is within the flight controller discipline level. For instance, modeling may occur within the EVA group or within the Flight Medical team. Each team must review the scheduled plan and ensure that the plan is feasible and meets the planning constraints. 


\section{Integrating Human Performance Measures into SPaCe Operations}

The following section proposes how human performance measures and modeling could potentially be better integrated into future space operations for exploration-class missions. For each process identified in Figure 1, methods will be presented where human performance measures may be included. Where appropriate, shortcomings related to existing planning and scheduling capabilities will be identified. Generally, human performance measures can be applied to state inputs (in this case, the astronaut's state) and to modeling human performance operational constraints \& operational objectives (i.e., assigned activities) with parameters that are relevant to human performance measures.

\section{State of Crew}

From Figure 1, one of the key external inputs to planning is state input. For human performance, this means providing the planning and scheduling tools with the current (or expected) state of crewmembers. This state of crewmember could be described as his or her overall readiness to perform assigned tasks; alternatively, it could be the state "at which skilled performance is most likely to be maintained at acceptable levels." [27, pp. A4] Part of this state would necessarily include an astronaut's wakefulness or alertness, which is based on amount of sleep, circadian rhythms, and sleep inertia [23, 26]. Disruptions in sleep have been known to decrease cognitive and neurobehavioral functions, such as vigilant attention, psychomotor and perceptual cognitive speed, and working memory [24]. With respect to planning and scheduling, this state (alongside human performance models) needs to be a predictor for the expected performance on an astronaut's assigned activity. Unfortunately, currently, the overall effect of decreased cognitive function on complex cognitive tasks (such as those activities scheduled during space operations) has not been thoroughly studied on Earth [24] or in spaceflight [7].

Fatigue also plays an important role with respect to describing a crew's readiness to perform. Tasks with high physical and/or cognitive workload add to a crewmember's fatigue. In order to better integrate the state of the crew into planning and scheduling tools, an astronaut's expected fatigue after completing assigned activities is needed. In the current planning process, there are planning constraints that account for fatigue. For instance, astronauts are given additional rest periods around busy and demanding events, such as EVAs and visiting vehicles [6].

In the future, other factors could weigh in on crew's state. These factors that may affect performance are currently still being researched, such as cumulative exposure to carbon dioxide and radiation [7]. Exploration class missions may also have to take into account the influence of gravitational changes or dust exposure as other environmental sources that may affect crew performance. For example, exploration missions to Mars may have to include planning constraints that minimize high physical tasks until astronaut has adapted to one-third gravity after long duration microgravity exposure on-route.

Part of a crew's overall readiness to perform has to include training. Astronauts go through extensive training on Earth before flight, which is also complemented in-flight for critical operations. For EVA and robotic tasks (i.e., manipulating ISS robotic arm), crew must complete onboard refreshers, which are scheduled for astronauts prior to the event $[8,9,10]$. For other activities, flight controllers need to verify that the crewmembers have completed the required training associated with a scheduled activity, i.e., crew can only be assigned trained tasks [6]. For explorationclass missions, verifying required training against scheduled, assigned activities will be critical as the training paradigm for this mission class will shift to increased onboard training [11]. Planning and scheduling tools can verify if the prerequisite training has already been completed by the assigned crewmember. Furthermore, temporal relationships could be incorporated in the scheduling tool to describe the minimum time between onboard, refresher training and scheduled activities (assuming the expected training retention of the task was modeled as a function of time).

Current planning and scheduling software capabilities allow for digitized ISS inputs. Flight controllers receive updates about ISS state variables (e.g., actual attitude vs. predicted) to inform replanning. Similarly, crew readiness to perform state could be an input into planning and scheduling tools and used as inputs for integrated human performance models of operations. This would require collection and incorporation of real-time measures of human performance and/or factors that influence performance. For instance, pertinent measures include actual sleep quality, increases in radiation or $\mathrm{CO}_{2}$ exposure, measures of alertness, circadian rhythm measures, and newly trained tasks. These measures could then be leveraged in a planning and scheduling tool that integrated human performance models. If a crewmember becomes sick or incapacitated, that information would also inform planning decisions.

\section{Modeling of Operations and Objectives}

Modeling of operations and objectives (see Figure 1) for crewmembers can be summarized as activity or task modeling. The tasks astronauts must complete and how they are executed is, in essence, what must be modeled, planned, and ultimately, scheduled. As previously mentioned, some task modeling is already conducted. With respect to human performance, time to completion is the main measure modeled. Planning task duration of activities is currently very conservative; activity durations are estimated based on procedure completion times multiplied by 1.4 to account for microgravity [12]. Additionally, task completion times are rounded to the nearest 5-minute in order to facilitate planning and scheduling [6]. 
If integrating task modeling into planning and scheduling tools, there are only a few options with regards to predicting task performance: estimate performance based on previous experiences, use measured ground-based task performance, or predict task performance based on human performance models. Even if just considering task completion times as the main human performance measure, predicting crew time is currently challenging. Modeling task performance cannot be agnostic to system effects. Performance is affected by the interface used (e.g., its saliency \& transparency), reliability, and automation type [13], not to mention the effectiveness of training for that activity and associated procedures' design. Some tasks might be done more efficiently earlier in the day, necessitating modeling them as a function of timeof-day [15]. Finally, task performance may be influenced by the order of tasks (e.g., monotony effect [15], task tempo [14]) and the effect of having multiple crewmembers work on a task. Some tasks may be completed by one crewmember at a time, but it might be more time effective for two astronauts to execute the task.

Updating models of operation and objectives requires collecting operational data as evidence for discrepancies, validating suggested modeling updates, and finally, implementing those changes in planning and scheduling software. While conceptually straightforward, it requires a long lead-time, as these updates are typically not easy to implement on software that has been certified for space operations, for which planning and scheduling tools are no exception. Facilitating the update process will be beneficial for all flight controller disciplines, including an integrated human performance modeling for planning and scheduling. For instance, future task modeling could be updated based on tracked task on time and other performance measures. This could, in particular, realize incorporating individual performance differences. Tracking the number of times a crewmember has completed certain activity may also allow inclusion of modeling task expertise development over the course of a mission.

\section{Operational Constraints}

If human performance modeling is to be seamlessly integrated into planning and scheduling tool alongside all other planning modeling and constraints, crew-driven operational constraints need to be also modeled. Most of these constraints that focus on crew-driven planning constraints are captured in the GGr\&C, Part 2 [6]. As previously mentioned, Ops Planners verify that crew are assigned the maximum planned hours of work-hours per day. If the maximum hours are exceeded, Score (planning and scheduling tool for crew planning) identifies that the constraint is not met, and shows a violation (see Figure 3 for visual example).

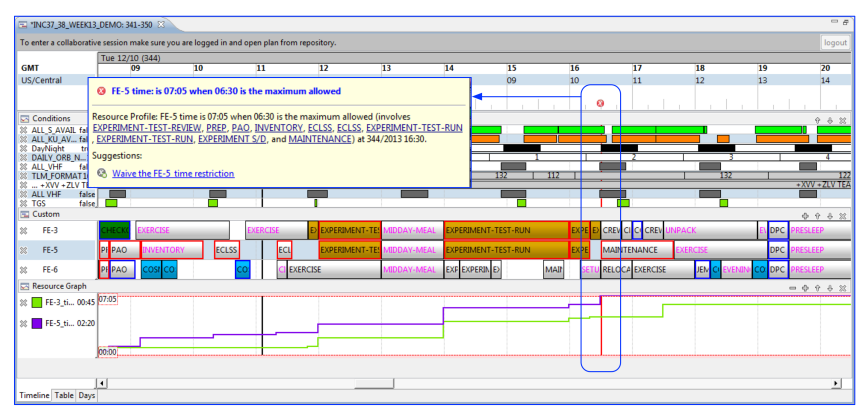

Figure 3: Score visualization of crew-driven operational constraint violation warnings

Other examples of crew-driven constraints are the number of rest days required or the exact number of sleep hours that should be planned. The flight controller team discusses and verifies that those planning constraints are met based on an initially proposed timeline. Similarly, if a sleep shift is needed (based on an event like docking of a visiting vehicle), the planning team and medical team work together to come up with an integrated sleep shift proposal. However, the planning constraint is not modeled per se within Score. Such a model would facilitate in the development of an initial timeline for flight controllers to discuss.

There are other crew-driven operational constraints that are not explicitly modeled as human performance constraints but simply rather as planning constraints. One example of this is the planning constraints regarding exercise. Crewmembers are required to exercise for two hours and thirty minutes each day; this is a crew-driven operational constraint. However, on EVA days, the EVA crews are not required to exercise [6]. The rationale for this planning constraint should be fairly straightforward: EVAs take up a large portion of the workday and are physically taxing to crewmembers; hence, exercising is not required on those days. In order to model this planning constraint as a crewdriven operational constraint, the emphasis would be on the number of hours a crewmember ought to perform physical exercise within a 24-hour day span. While subtle, it is an important distinction in terms of integrating human performance measures into planning and scheduling. For example, the crew-driven constraint could be stated as: every crewmember must be scheduled for at least 2.5 hours but not more than 8 hours of high-intensity physical workload within a 24-hour day span. In this latter modeling paradigm, each activity (such as an EVA) has some modeling related to physical workload it imposes on astronauts and in turn, there is a threshold on time (duration) and a threshold that defines high-intensity.

Potentially, there are many more crew-driven operational constraints that are temporal in nature, such as maximum cumulative radiation exposure. Current planning and scheduling tools are well suited to track this information and indicate when approaching set limits. Other temporal, crewdriven operational constraints may be envisioned for future integrated human performance modeling. For instance, there 
may be activities that require high cognitive workload and it may be undesirable to schedule astronauts for too many of these within some set timeframe [15]. In order to integrate such a crew-driven operational constraint, one must understand how to model activities with respect to cognitive workload, understand the state of crewmembers to workload capacity, and determine a maximum threshold for cognitive workload. A more complex crew-driven planning constraint example for planetary missions is determining the necessary time between EVAs to physically recover while also meeting cumulative environment exposure limits, such as those that would exist for radiation or dust.

\section{DisCUSSION}

The first part of this paper described ISS planning and scheduling capabilities. Many of the planning constraints for crew are resolved by the Ops Planner flight controller discipline, as they are responsible for integrating all inputs from other controllers into one shared plan. From the integrated, shared plan, the mission control flight controller team must verify that their specific planning constraints, be it related to crew or not, are addressed. Based on the existing planning and scheduling process, including existing modeling and supporting software tools, the second part of this paper proposes a method to integrate human performance measures into present support tools.

The underlying question for this paper is whether the existing planning and scheduling tools are limiting our ability to integrate human performance measures into space operations. Primarily, existing planning and scheduling tools do not prevent such integration, but there are several factors that do limit the extent in which human performance measures and modeling can be integrated.

While it is possible that the author is not aware of additional planning tools accessible to other flight controller disciplines, crew planning and scheduling is currently not fully integrated - meaning that the various flight controllers that verify that vehicle, scientific, and crew constraints are met - do not visualize, resolve, and interrelate these constraints in one place. It is difficult and in turn, timeconsuming, to understand the effect of one change in the schedule has on all operational constraints. This lack of integration, which would not be exclusive to incorporating human performance modeling, is one current limitation.

Currently, initial states of the vehicle (e.g., attitude, communication availability) are inputs to planning and scheduling tools, and these states are propagated based on existing operations modeling. In order to better integrate human performance modeling into space operations, one has to first address the important question: is it sufficient to predict crew state (and initial state) based on planned activities or based on real-time measures. Either choice presents future challenges in planning and scheduling performance modeling.
If crew state is based on planned activities, each task or activity the crew must complete needs to be modeled. For instance, how much cognitive workload is predicted for each different type of activity? Is it a function of the type of interface used? Does the number of crew supporting the activity affect predicted cognitive workload? How does it change when preceded by other types of activities? Such modeling of objectives would be a tremendous undertaking, not only for space operations planning but also for space human factors engineering. While there are advances in human performance modeling in some areas $[16,17]$, there are many components that are yet to be integrated and validated for space human flight. An integrated method to model crew activities would take into account system effects (e.g., type of automation), behavioral effects (e.g., stress), individual differences (e.g., training) and environmental effects (e.g., radiation exposure, light countermeasures).

Task modeling is a key component of human performance modeling (HPM) [16]. In the HPM domain, the task is described in a task network model. A specific task is modeled through sub-task components and the sequence, selection rules, and completion time of each subtask is defined [22]. Sub-tasks typically are at the granularity of "look at altimeter" or "decide if there is enough fuel." On the other hand, in space operations planning, tasks are described as activities, such as "conduct glove box experiment." At the procedure level, procedure steps are described in action, e.g., "open box" or "set light exposure".

In order to use existing ISS planning and scheduling tools, the granularity in which tasks are modeled would have to significantly change in order to incorporate human performance measures and modeling. In space operations, tasks are modeled in terms of duration, some requirements (e.g., occupies particular exercise machine or consumes certain amount of power), and type of activity. One key gap between operations and research is to better understand the categorizations already used in operations, relate them modeling attributes, and identify the missing modeling parameters that describe a task sufficiently. This would allow activities to be characterized "on the fly", i.e., new types tasks can easily be created and modeled. Arguably, such modeling would be an indispensible prerequisite for human performance modeling of future exploration tasks.

Task modeling based on planned activities also has the inherent problem that scheduled activities do not always go as planned. Sleep is a critical example. While ISS astronauts are scheduled for eight hours of sleep, research indicates that crews experience sleep loss [25]. Should planning reflect scheduled or actual sleep or sleep quality? How would chronic partial sleep by represented? If planning and scheduling incorporate on-orbit performance measures, the challenge becomes unobtrusively measuring and tracking the appropriate metrics. This would also be a significant shift in operations, as currently crew 
performance measures are not systematically tracked [12], though there are recent efforts to begin addressing this [18].

Tracking human performance measures in real-time would help to improve and update performance and task models, resulting in future predictions that are more accurate, and hence, more efficiently scheduled. Noteworthy, most planning software tools do not automatically take in realtime inputs as they are primarily used as predictive tools. Real-time tracking may also help plan for cumulative effects. Planning and scheduling tools could easily support tracking of cumulative effects but would have a more difficult time characterizing it as detailed ISS modeling starts a week ahead [12] and plans are only modified right up to the day before execution.

Another relevant human performance measure that has effects longer than a week-at-a-time is fatigue due to chronic sleep deficiencies. If considering integration into operations, alertness may be a better operational metric for planning rather than cumulative sleep quality. Arguably, crewmembers may be the ultimate "real-time tracker" of their readiness to perform, creating the opportunity for dayof replanning or self-scheduling. Previously, researchers have suggested that crew should have greater flexibility in determining their schedule [15]. It is important to understand which types of tasks and under which circumstances self-scheduling is appropriate during space operations, as there is evidence that people are poor estimators of their alertness [23].

Planning and scheduling for space operations is predicated by the fact that there are many operational constraints that need to be satisfied, and moreover, that each of these has a threshold. Not meeting those thresholds results in a violation that needs to be resolved by some change in schedule. When it comes to human performance, current space operations have defined constraints and rules to protect crew (as for example, delineated in the GGr\&C). Future human spaceflight programs will depend on the NASA Standards $3001[19,20]$ to provide guidance with regards to human performance limits. These standards are meant to support the development of future concepts of operations, mission operations documentation, and program documents and requirements.

From the perspective of the operational capabilities planning and scheduling software tools have, we need human performance thresholds that are based on integrated task and schedule modeling. NASA Standards 3001 Vol. 1 and Vol. 2 provides several standards that support this need (Table 1). However, the NASA Human Integration Design Handbook (HIDH) [21], while it describes human performance measures and suggested methods for measurement, it only hints at some thresholds. The recommended thresholds are not tied to task type nor validated beyond laboratory settings. Future space mission operations will need NASA Standards that further refine human performance thresholds if human performance modeling is to be integrated into planning and scheduling tools.

Table 1: Selected NASA standards related to integrated human performance $[19,20]$

\begin{tabular}{|c|c|}
\hline $\begin{array}{l}\text { NASA } \\
\text { Standard }\end{array}$ & Standard Description \\
\hline 4.2.4.3 & $\begin{array}{l}\text { Fitness-for-Duty } \\
\text { Standard: In-flight Fitness-for-Duty } \\
\text { standards shall be assessed using } \\
\text { metrics that are task specific. }\end{array}$ \\
\hline 4.2 .5 .4 & $\begin{array}{l}\text { Fitness-for-Duty Behavioral Health } \\
\text { and Cognition Standard: The planned } \\
\text { number of hours for completion of } \\
\text { critical tasks and events, workday, } \\
\text { and planned sleep period shall have } \\
\text { established limits to assure continued } \\
\text { crew health and safety. }\end{array}$ \\
\hline 4.4.2.5 & $\begin{array}{l}\text { Circadian Shifting Operations and } \\
\text { Fatigue Management: Support of crew } \\
\text { schedule planning and operations } \\
\text { shall be provided to include circadian } \\
\text { entrainment, work/rest schedule } \\
\text { assessment, task loading assessment, } \\
\text { countermeasures, and input to special } \\
\text { activity schedules. }\end{array}$ \\
\hline 4.4 .3 .5 .2 & $\begin{array}{l}\text { General Health and Well Being: } \\
\text { Countermeasures shall be provided to } \\
\text { address issues of human factors and } \\
\text { general crew health and well-being, } \\
\text { including considerations for hygiene, } \\
\text { privacy, nutrition, crew schedule, } \\
\text { workload, Earth observation, and } \\
\text { leisure activities. }\end{array}$ \\
\hline 5.2 .1 & $\begin{array}{l}\text { Time and Performance: The ability to } \\
\text { perform tasks in a timely and } \\
\text { accurate manner shall be } \\
\text { accommodated in the design of all } \\
\text { system elements that interface with } \\
\text { the crew for all levels of crew } \\
\text { capability and all levels of task } \\
\text { demands. }\end{array}$ \\
\hline 5.2 .2 & $\begin{array}{l}\text { Situational Awareness (SA): Systems } \\
\text { shall be designed such that the SA } \\
\text { necessary for efficient and effective } \\
\text { task performance is provided and } \\
\text { can be maintained for all levels of } \\
\text { crew capability and all levels of task } \\
\text { demands. }\end{array}$ \\
\hline 5.2 .3 & $\begin{array}{l}\text { Cognitive Workload: Cognitive } \\
\text { workload shall be accommodated (to } \\
\text { avoid overload or underload) in the } \\
\text { design of all system elements that } \\
\text { interface with the crew for all levels } \\
\text { of crew capability and all levels of } \\
\text { task demands. }\end{array}$ \\
\hline
\end{tabular}




\section{CONCLUSION}

ISS planning and scheduling is a critical centerpiece of space operations. There are several planning and scheduling software tools that help flight controllers model ISS activities, resources, and constraints. Currently, human performance measures and modeling is not tightly integrated into these planning and scheduling tools. Ideally, all vehicle and crew planning would be integrated, allowing planners to understand the complexity and effects one change in the plan has on the whole system. This paper proposes, based on the existing capabilities of these planning tools, how this integration could be possible, identifying gaps between planning software architectures and human performance modeling. If integration is desired, future work should focus on modeling crew tasks at the activity level as well as determining appropriate human performance limits for planning and scheduling space operations.

\section{REFERENCES}

[1] Marquez, J. J. (2007) Human-Automation Collaboration: Decision Support for Lunar and Planetary Exploration (Doctoral Dissertation). Massachusetts Institute of Technology, Cambridge, MA.

[2] Cummings, M. L., Marquez, J. J., Roy, N. (2012) "Human-automated path planning optimization and decision support." International Journal of HumanComputer Studies, Volume 70(2), pp. 116-128.

[3] Blau, P. (2014, May 12) ISS suffers Partial Loss of Power - Mission Control evaluates Recovery Options. Retrieved from http://www.spaceflight101.com

[4] Frank, J. (2010) "When plans are executed by mice and men." Aerospace Conference, 2010 IEEE, March 2010.

[5] Smith, E.E., Korsmeyer, D.J., Hall, V.F., Marquez, J., Iverson, D., Trimble, J. et al (2014) "Exploration Technologies for Operations." SpaceOps 2014 Conference, May 2014.

[6] NASA (2012) ISS Generic Groundrules and Constraints Part 2: Execute Planning. International Space Station Program, SSP 50261-02, Rev. D. Houston, TX.

[7] Strangman, G. \& Beven, G. (2013) "Review of Human Cognitive Performance in Spaceflight." Presentation at 84th Annual Scientific Meeting of the Aerospace Medical Association, May 2013.

[8] Johnson, G. and Alexander, G. (2013) "Robotics OnBoard Trainer (ROBoT)" NASA Tech Briefs, April 2013.

[9] NASA (2014, April 15) "ISS Daily Summary Report 04/15/14" [Web log post]. Retrieved October 15, 2014, from http://blogs.nasa.gov/stationreport.
[10] Gerst, A. (2014, October 5) [Web microblog post] Retrieved October 15, 2014, from https://twitter.com/Astro_Alex/status/5187931676349849 63.

[11] Barshi, I. (2012) Evidence Report: Risk of Performance Errors due to Training Deficiencies. Houston, TX: NASA Johnson Space Center. Retrieved from http://humanresearchroadmap.nasa.gov

[12] Clement, G. (2011) Fundamentals of Space Medicine (2nd ed.) New York, NY: Springer.

[13] Sebok, A., Wickens, C., Clegg, B. \& Sargent, R. (2014) "Using Empirical Research and Computational Modeling to Predict Operator Response to Unexpected Events." Paper presented in 2014 International Annual Meeting of Human Factors and Ergonomics Society. October 2014.

[14] Sandor, A., Schuh, S.V., \& Gore, B. F. (2013) Evidence Report: Risk of Inadequate Critical Task Design. Houston, TX: NASA Johnson Space Center. Retrieved from http://humanresearchroadmap.nasa.gov.

[15] Connors, M. M., Harrison, A. A., \& Akins, F.R. (1985) Living aloft: Human requirements for extended spaceflight. Washington, DC: Scientific and Technical Information Branch, NASA.

[16] Gore, B.F. \& Smith, J.D. (2006) "Risk assessment and human performance modelling: the need for an integrated systems approach." International Journal of Human Factors Modelling and Simulation, Vol. 1(1), pp. 119139.

[17] Wickens, C., Sebok, A., Keller, J., Peters, S., Small, S. Hutchins, S. et al (2013) Modeling and Evaluating Pilot Performance in NextGen: Review of and Recommendations Regarding Pilot Modeling Efforts, Architectures, and Validation Studies (NASA Technical Memorandum-2013-216504). Moffett Field, CA: NASA Ames Research Center.

[18] Human Performance Data Project (2014, July 30). Retrieved from http://humanresearchroadmap.nasa.gov/tasks/?i=1803.

[19] NASA (2014) NASA Space Flight Human-System Standard, Volume 1, Revision A: Crew Health (NASASTD-3001, Vol. 1). Washington, DC: NASA.

[20] NASA (2011) NASA Space Flight Human-System Standard, Volume 2: Human Factors, Habitability, and Environmental Health (NASA-STD-3001, Vol. 2). Washington, DC: NASA.

[21] NASA (2010) Human Integration Design Handbook (HIDH) (NASA/SP-2010-3407). Washington, DC: NASA. 
[22] Fallon, E.F. (2006) "Computer Simulation". International Encyclopedia of Ergonomics and Human Factors (2nd ed.). CRC Press.

[23] Mallis, M.M. \& DeRoshia, C.W. (2005) "Circadian Rhythms, Sleep, and Performance." Aviation, Space, and Environmental Medicine, Vol 76(6), pp. B94-B107,

[24] Goel, N., Basner, M., Rao, H., \& Dinges, D.F. (2013) "Chapter Seven - Circadian Rhythms, Sleep Deprivation, and Human Performance" Progress in Molecular Biology and Translational Science, Vol. 119, pp. 155-190.

[25] Barger, L.K., Flynn-Evans, E.E., Kubey, A., Walsh, L., Rondan, J.M., Wang, W., et al (2014) "Prevalence of sleep deficiency and use of hypnotic drugs in astronauts before, during, and after spaceflight: an observational study." The Lancet Neurology, Vol. 13(9), pp. 904-912.

[26] Van Dongen, H.P.A. (2004) "Comparison of Mathematical Model Predictions to Experimental Data of Fatigue and Performance." Aviation, Space, and Environmental Medicine, Vol. 75(3), Section II, pp. A15A36.

[27] Mallis, M.M., Mejdal, S., Nguyen, T.T., \& Dinges, D.F. (2004) "Summary of the Key Features of Seven Biomathematical Models of Human Fatigues and Performance." Aviation, Space, and Environmental Medicine, Vol. 75(3), Section II, pp. A4-A14.

\section{BIOGRAPHY}

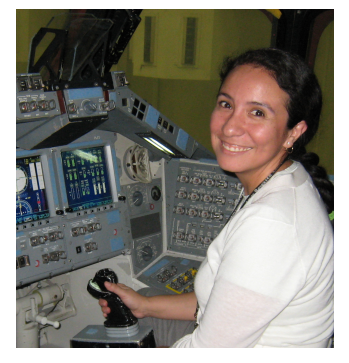

Jessica J. Marquez received a B.S.E. in Mechanical Engineering from Princeton University, followed by a S.M. from the Department of Aeronautics and Astronautics at the Massachusetts Institute of Technology. She received her Ph.D. in Human Systems

Engineering from the Massachusetts Institute of Technology. Since 2007, she has been working at the NASA Ames Research Center within the Human Systems Integration Division. As part of the Human Computer Interaction Group, she has supported the development and deployment of various instances of SPIFe (Scheduling \& Planning Interface For exploration), the planning and scheduling software tool for several space missions, leading the PLATO and Score projects for International Space Station. She continues to conduct research in the field of human-automation integration, human-computer interaction, and space human factors engineering. 